\title{
Degradação de Poliolefinas Utilizando Catalisadores Zeolíticos
}

\author{
Maria Letícia M. Valle, Maria José 0. C. Guimarães \\ Departamento de Processos Orgânicos, EQ, UFRJ \\ Cristiane M. S. Sampaio \\ INMETRO, RJ
}

\begin{abstract}
Resumo: Neste trabalho foi estudada a degradação de alguns dos principais constituintes dos rejeitos plásticos (polietileno de alta densidade (HDPE), polietileno de baixa densidade (LDPE) e polipropileno (PP)), empregando-se um catalisador exausto de unidades de craqueamento de fluidos (FCC) e um catalisador zeolítico (ADZ3) sintetizado em laboratório. Utilizando técnicas de termogravimetria (TG-DTG) e cromatografia gasosa (CG), foi possível avaliar os produtos gerados no craqueamento destas poliolefinas. Na degradação catalítica de poliolefinas com catalisadores zeolíticos, verificouse a obtenção preferencial de gasolina, GLP e diesel, produtos importantes na matriz energética brasileira. O catalisador de FCC exausto foi mais seletivo para a produção de gasolina e GLP, enquanto que a produção de diesel foi mais favorecida com o catalisador ADZ3.
\end{abstract}

Palavras-chave: Poliolefinas, degradação, análise térmica, frações combustíveis, zeólitas.

\section{Degradation of Polyolefins Using Zeolitic Catalysts}

Abstract: In this work the degradation of some of the main plastics responsible for waste, viz. high density polyethylene (HDPE), low density polyethylene (LDPE) and polypropylene (PP), was studied using a spent FCC catalyst (fluid cracking catalyst) and a zeolitic catalyst (ADZ3) synthesized in laboratory. Using thermogravimetry (TG-DTG) and gas chromatography (GC) techniques, it was possible to evaluate the products from these polyolefins cracking. The catalytic degradation of polyolefins led to a preferential production of LPG, diesel and gasoline, which are important products of the Brazilian energetic matrix. The spent FCC catalyst was more selective for production of LPG and gasoline, whereas the diesel production was more favored with the ADZ3 catalyst.

Keywords: Polyolefins, degradation, thermal analysis, fuels, zeolites.

\section{Introdução}

Nas últimas décadas, considerável quantidade de pesquisa e desenvolvimento tem sido realizada, com a finalidade de se obter combustíveis e matérias-primas a partir de rejeitos plásticos. Essas pesquisas visam a necessidade de se minimizar o problema do acúmulo do lixo sólido, cujos rejeitos plásticos podem ser convertidos, via degradação térmica e/ou catalítica, em produtos com elevado valor $\operatorname{agregado}^{[1,2]}$.

Estados Unidos, Japão e China são os países que mais têm investido em pesquisa nessa área, destacando-se a China em número de patentes depositadas ${ }^{[1]}$. A degradação de polímeros tem se tornado um método crescente e importante, para converter rejeitos plásticos em produtos químicos, ou seja, em frações líquidas usadas como combustível ou destinadas ao setor petroquímico ${ }^{[3-6]}$.

O craqueamento de polímeros requer um sistema catalítico eficiente, envolvendo um conhecimento detalha- do das relações entre as propriedades do catalisador e a distribuição dos produtos formados. A utilização de catalisadores contendo sítios ácidos de Lewis e de Brönsted tem possibilitado um maior controle dessa distribuição no craqueamento dos polímeros ${ }^{[7-10]}$. Uddin e colaboradores comparando a degradação de diferentes polietilenos na presença ou ausência de sílica-alumina, verificaram uma diferença significativa na distribuição dos produtos ${ }^{[3]}$. A presença de sílica-alumina inibiu a formação de compostos pesados $\left(>\mathrm{C}_{22}\right)$ e maximizou a produção de gasolina $\left(\mathrm{C}_{5}-\mathrm{C}_{12}\right)$. Desse modo, os catalisadores zeolíticos aparecem como fonte potencial para o craqueamento catalítico de polí$\operatorname{meros}^{[4,8,10,11]}$.

O estudo do processo de craqueamento catalítico de polímeros é altamente complexo, em decorrência do grande número de reações envolvidas, as quais são específicas para cada tipo de polímero. A modelagem do craqueamento catalítico tem atraído a atenção de muitos pesquisadores e associa, com freqüência, dados de análise térmica (TG) 
em complemento a experimentos conduzidos em reatores catalíticos $^{[6,8-12]}$.

No craqueamento de polímeros os custos associados ao sistema catalítico podem ser minimizados, pela reutilização de rejeitos de catalisadores de FCC de unidades de craqueamento de fluidos ${ }^{[13,14]}$. Estes catalisadores, oriundos de refinarias de petróleo, possuem ainda boa atividade catalítica, podendo ser empregados em outros tipos de processo. O catalisador de FCC é constituído de uma matriz (alumina), de um ligante (ślica) e de uma zeólita Y trocada em geral com terras raras ${ }^{[15]}$.

Neste trabalho foi estudada a degradação catalítica de polietileno de alta e baixa densidade e polipropileno, utilizando uma zeólita sintetizada em laboratório (ADZ3) e um catalisador de FCC oriundo de uma refinaria de petróleo. A distribuição dos produtos formados foi analisada empregando-se técnicas combinadas de análise térmica e cromatografia gasosa.

\section{Experimental}

\section{Materiais e Métodos}

A Tabela 1 apresenta as especificações dos polímeros utilizados neste trabalho. Os polímeros foram tratados por um processo de solubilização/precipitação para obtenção dos mesmos em forma de pó. Tolueno a $90{ }^{\circ} \mathrm{C}$ e triclorobenzeno a $135^{\circ} \mathrm{C}$ foram empregados para solubilizar os polietilenos e o polipropileno respectivamente. Os polímeros precipitados com metanol foram filtrados à vácuo e secos em estufa a $80^{\circ} \mathrm{C}$ até peso constante. Para o craqueamento catalítico foram empregados um catalisador exausto de FCC doado pelo CENPES/PETROBRAS, e uma zeólita ADZ3 sintetizada em laboratório ${ }^{[16]}$. A ativação dos catalisadores foi realizada em estufa à vácuo a $120^{\circ} \mathrm{C}$ por 8 horas.

O processo de craqueamento foi conduzido em equipamento TG-DTG, modelo SDT 2860 da TA Instruments, sob vazão de nitrogênio de $100 \mathrm{ml} / \mathrm{min}$, taxa de aquecimento de $10{ }^{\circ} \mathrm{C} / \mathrm{min}$ e faixa de temperatura de $30{ }^{\circ} \mathrm{C}$ a $800{ }^{\circ} \mathrm{C}$. Foram empregadas percentagens mássicas de catalisadores de $10 \%, 25 \%$ e $50 \%$. As temperaturas iniciais $\left(\mathrm{T}_{\text {onset }}\right)$ e finais $\left(\mathrm{T}_{\mathrm{f}}\right)$ de degradação dos polímeros foram determinadas, segundo metodologia descrita por Hatakeyama e colaboradores ${ }^{[17]}$, utilizando as curvas de TG e o Programa Universal Analysis, versão 2.6D, da TA Instruments. As temperaturas máximas $\left(\mathrm{T}_{\text {máx }}\right)$ de degradação foram calculadas a partir das curvas de DTG.

\section{Caracterização da distribuição de produtos}

Os gases efluentes do termoanalisador SDT 2860 foram

Tabela 1. Especificações das Poliolefinas

\begin{tabular}{ccccc}
\hline Polímero & Produtor & Mn* & Mw* & Polidispersão \\
\hline LDPE & POLIOLEFINAS & 52.260 & 215.850 & 4,13 \\
HDPE & POLIALDEN & 28.367 & 97.040 & 2,52 \\
PP & POLIBRASIL & 88.570 & 246.330 & 2,78 \\
\hline
\end{tabular}

* Determinados por SEC (triclorobenzeno, $140^{\circ} \mathrm{C}$ ) coletados com auxílio de uma seringa para gases e analisados em cromatógrafo a gás Agilent 6890, equipado com coluna HP $1(30 \mathrm{~mm}$ X $0,25 \mathrm{~mm}$ X $0,25 \mu \mathrm{m})$ e detetor de ionização de chama FID. O início da coleta dos gases correspondeu a $20 \%$ de degradação em relação à massa de polímero e o término a $30 \%$. Os gases do craqueamento foram agrupados em frações segundo critérios estabelecidos nas refinarias de petróleo: gás combustível $\left(\mathrm{C}_{1}-\mathrm{C}_{2}\right)$, GLP $\left(\mathrm{C}_{3}-\mathrm{C}_{4}\right)$, gasolina $\left(\mathrm{C}_{5}-\mathrm{C}_{12}\right)$, diesel $\left(\mathrm{C}_{12}-\mathrm{C}_{22}\right)$ e pesados $\left(>\mathrm{C}_{22}\right)$. A faixa dos tempos de retenção das várias frações foi determinada utilizando misturas de hidrocarbonetos padrões e o Programa GC Chemstation Agilent Technologies, conforme metodologia descrita na referência [2].

\section{Resultados e Discussão}

\section{Influência do tipo de catalisador nas temperaturas de degradação}

A Tabela 2 apresenta as análises termogravimétricas das poliolefinas puras e de suas misturas com $50 \%$ de catalisador.

Os catalisadores atuam de forma significativa na redução das principais temperaturas de degradação. Considerando a redução da temperatura inicial de degradação como uma medida da eficiência catalítica, verifica-se como esperado, que o catalisador ADZ3 foi mais ativo que o de FCC. A maior redução da temperatura inicial de degradação foi observada para o HDPE, indicando uma maior eficiência dos catalisadores no craqueamento deste polímero. Tal fato pode ser possivelmente atribuído à linearidade da cadeia polimérica, e conseqüentemente, à facilidade de penetração dos fragmentos macromoleculares nos poros dos catalisadores zeolíticos. A menor eficiência foi observada para o LDPE seguido do PP. A presença de ramificações de cadeia curta e longa dificulta o acesso dessas macromoléculas aos poros dos catalisadores zeolíticos diminuindo assim a eficiência do processo de craqueamento. Resultados semelhantes foram também encontrados por outros pesquisadores para diferentes catalisadores zeolíticos ${ }^{[6,18]}$.

Tabela 2. Influência do tipo de catalisador na temperatura de degradação

\begin{tabular}{|c|c|c|c|c|c|}
\hline Polímeros & Catalisador & $\mathbf{T}_{\text {onset }}\left({ }^{\circ} \mathbf{C}\right)$ & $\mathbf{T}_{\mathbf{f}}\left({ }^{\circ} \mathbf{C}\right)$ & $\mathbf{T}_{\text {máx. }}\left({ }^{\circ} \mathbf{C}\right)$ & $\Delta \mathbf{T}_{\text {onset }}\left({ }^{\circ} \mathbf{C}\right)^{(1)}$ \\
\hline \multirow{3}{*}{ HDPE } & - & 460 & 493 & 482 & \\
\hline & ADZ3 & 206 & 313 & 240 & 254 \\
\hline & FCC & 324 & 403 & 376 & 136 \\
\hline \multirow{3}{*}{ LDPE } & - & 453 & 492 & 480 & \\
\hline & ADZ3 & 224 & 362 & 262 & 229 \\
\hline & FCC & 386 & 438 & 429 & 67 \\
\hline \multirow{3}{*}{ PP } & - & 426 & 471 & 457 & \\
\hline & ADZ3 & 190 & 248 & 217 & 236 \\
\hline & FCC & 306 & 368 & 349 & 120 \\
\hline
\end{tabular}


A Figura 1 apresenta as curvas de DTG para os polímeros puros e suas misturas catalíticas. As curvas de DTG, além de servirem como base para a determinação das temperaturas máximas de degradação dos polímeros, fornecem também

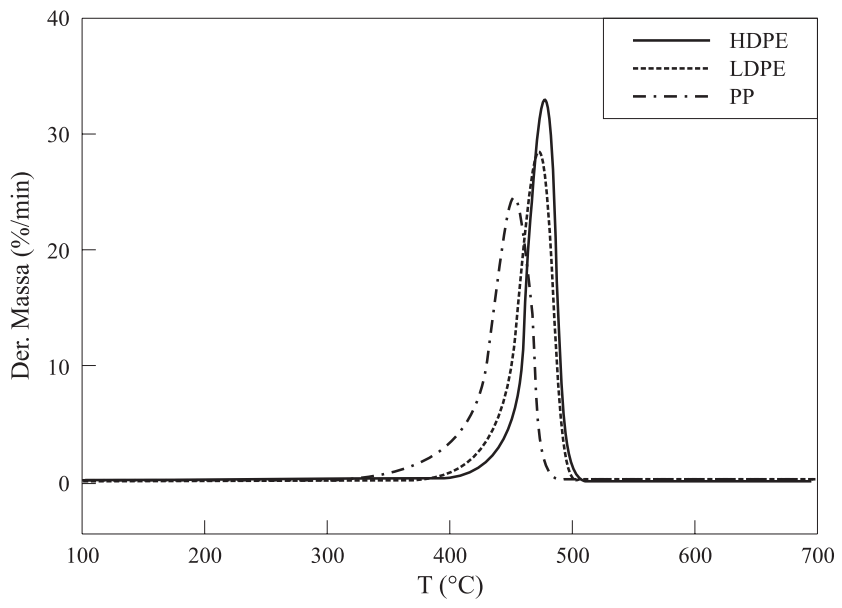

(a)

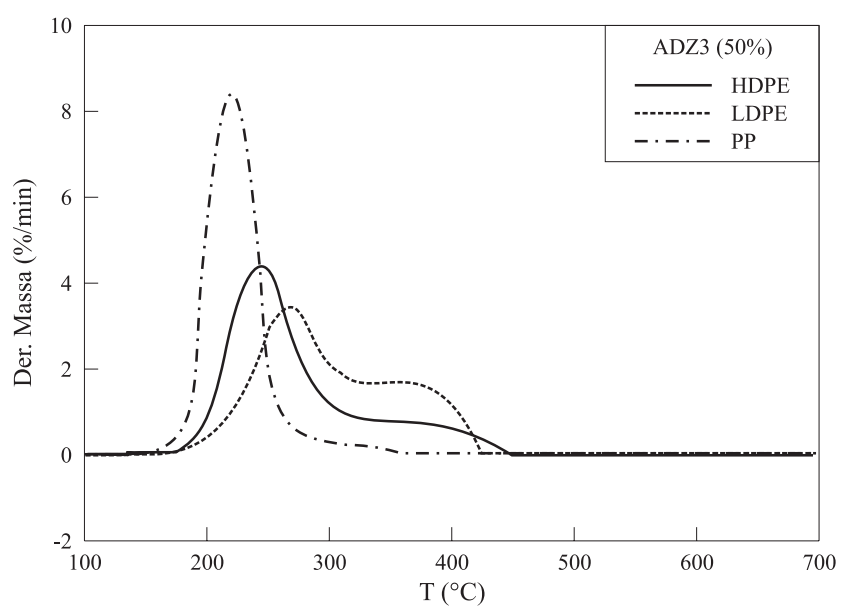

(b)

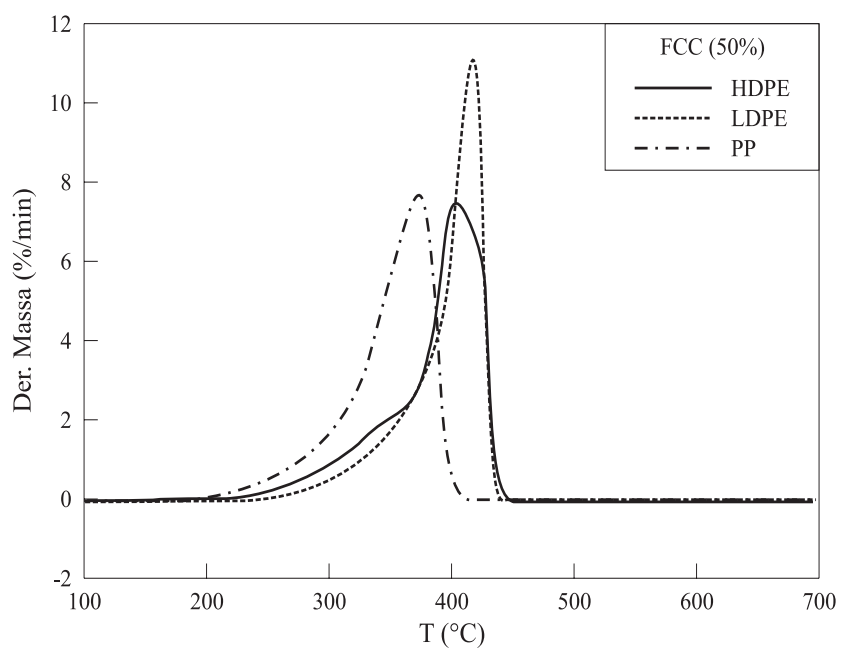

(c)

Figura 1. Curvas de DTG: (a) polímeros puros, (b) com ADZ3, (c) com FCC valiosas informações sobre os estágios e tipos de mecanismos operantes no processo de craqueamento catalítico.

Observa-se que para os polímeros puros, a degradação térmica ocorre em um único estágio. A degradação catalítica com a ADZ3 ocorre em um único estágio para o PP, e mais de um estágio para os polietilenos. Além da diferença na arquitetura macromolecular destes polímeros, outro fator responsável por este comportamento é a existência de diferentes tipos de sítios ácidos no catalisador ADZ3, os quais podem interagir com diferentes pontos de ruptura na cadeia macromolecular. Embora possua cadeia linear, o HDPE pode apresentar também alguns grupos laterais gerados por transferência de cadeia durante o processo de polimerização. Esses grupos formam ramificações de cadeia curta aleatoriamente distribuidas na cadeia do polímero e podem interagir com os diferentes sítios ácidos do catalisador. Comportamento semelhante é observado para o LDPE, sendo que este polímero além de apresentar ramificações de cadeia curta, também apresenta ramificações de cadeia longa distribuídas aleatoriamente, tornando-o bem mais heterogêneo e com uma propoção maior de centros de ruptura, conforme verificado pelo alargamento das curvas de DTG. O PP não apresenta ramificações em sua macromolécula, mas substituintes $\mathrm{CH}_{3}$ em cada unidade repetitiva, com uma mesma configuração ao longo da cadeia polimérica (polímero isotático). Assim, este polímero exibe um único centro de ruptura e, conseqüentemente, um único estágio de degradação.

As curvas de DTG para a degradação com o catalisador de FCC mostram também, um único estágio de degradação para o LDPE e o PP, e mais de um estágio para o HDPE. O catalisador de FCC exausto, devido à contaminação com coque e outras impurezas apresenta menor acessibilidade a seus diferentes centros ativos. Como conseqüência, os polímeros ramificados não conseguem ter acesso à todos os centros catalíticos, e mostram assim, comportamento semelhante ao observado no craqueamento térmico (ausência de catalisador). O HDPE devido à sua estrutura linear e após um pré-craqueamento na matriz, consegue ter um maior acesso aos diferentes sítios catalíticos.

A Tabela 3 mostra as energias de ativação para o processo de craqueamento segundo metodologia desenvolvida por Doyle ${ }^{[19]}$.

Os valores calculados para o craqueamento térmico do HDPE $(225 \mathrm{KJ} / \mathrm{mol})$, para o LDPE $(200 \mathrm{KJ} / \mathrm{mol})$ e para o

Tabela 3. Energias de ativação média dos processos de degradação dos polímeros

\begin{tabular}{lccc}
\hline \multirow{2}{*}{ Amostras } & \multicolumn{3}{c}{$\Delta \boldsymbol{E}(\mathbf{K J} / \mathbf{m o l})$} \\
\cline { 2 - 4 } & HDPE & LDPE & PP \\
\hline Polímeros puros & 225 & 200 & 212 \\
+ ADZ3 (50\%) & 96 & 133 & 137 \\
+ FCC exausto(50\%) & 89 & 112 & 118 \\
\hline
\end{tabular}

$\Delta \mathrm{E}=$ Energia de ativação média 
PP $(212 \mathrm{KJ} / \mathrm{mol})$, estão de acordo com os encontrados na literatura ${ }^{[19,20]}$. Uma redução significativa como esperado, é observada para o craqueamento catalítico dos polímeros ${ }^{[21,22]}$.

Apesar de não se conhecer a composição exata do catalisador de FCC exausto, provavelmente, sua maior atuação na redução da energia de ativação das poliolefinas, devese à presença de outras substâncias (matriz e ligante) em sua formulação, que promovem um pré-craqueamento das macromoléculas, e atuam assim em conjunto com a zeólita também presente.

\section{Caracterização da Distribuição dos Produtos}

A distribuição dos produtos do craqueamento das poliolefinas puras e de suas misturas com os catalisadores ADZ3 e FCC exausto, nas várias frações mássicas, é apresentada na Figura 2. O rendimento em gás combustível para todas as amostras foi próximo a zero, assim não é mostrado nos gráficos.

Para todos os polímeros observa-se, com ambos os catalisadores, um craqueamento preferencial em gasolina,

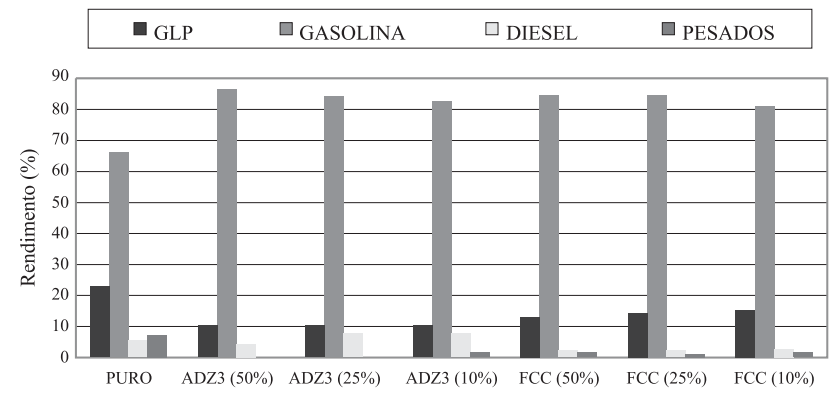

(a)

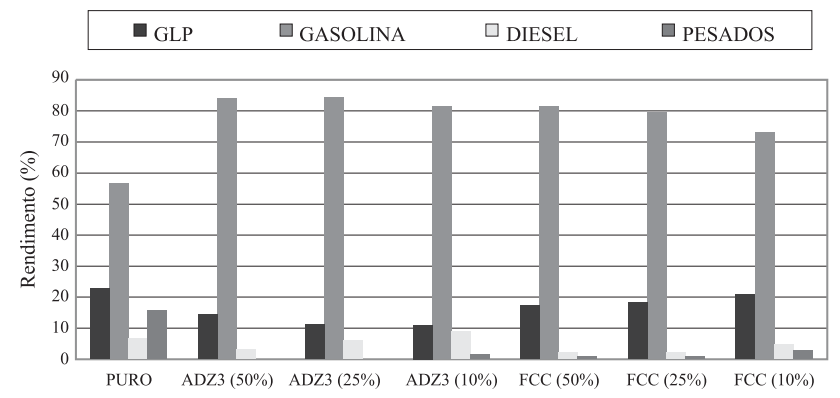

(b)

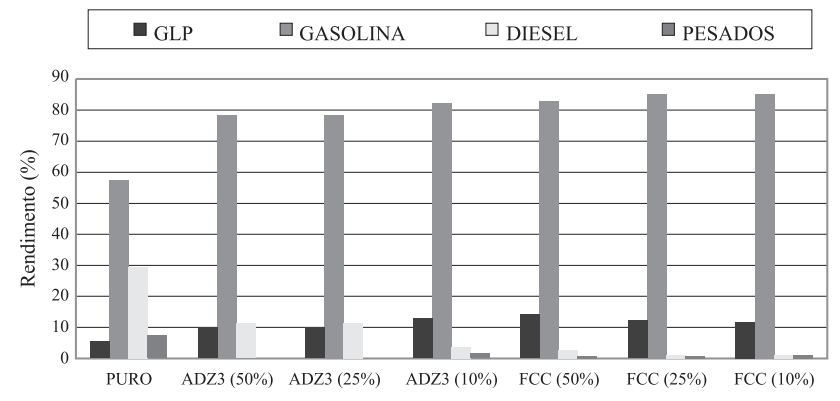

(c)

Figura 2. Distribuição de Produtos para o Processo de Craqueamento: (a) HDPE, (b) LDPE, (c) PP com rendimento praticamente independente da fração mássica de catalisador empregada. É possível verificar, também, que a presença dos catalisadores reduziu significantemente a produção de hidrocarbonetos pesados acima de $\mathrm{C}_{22}$ (ceras), similarmente ao encontrado por outros pesquisadores $^{[3,4,23,24]}$.

$\mathrm{O}$ craqueamento térmico do LDPE apresentou maior rendimento em pesados do que o do HDPE, o que pode ser atribuído à estrutura mais heterogênea do LDPE, indicando que a ruptura na cadeia macromolécular ocorre, provavelmente, nos pontos onde existem as ramificações de cadeia longa.

$\mathrm{O}$ craqueamento térmico do $\mathrm{PP}$ apresentou rendimento em pesados menor que o do LDPE e semelhante ao HDPE. O rendimento em diesel foi bem maior do que o apresentado pelos polietilenos. Resultado similar foi encontrado por Predel \& Kaminsky, ao realizarem, também, o craqueamento térmico destes polímeros ${ }^{[6]}$.

Basicamente, o catalisador de FCC exausto produziu dois produtos: GLP e gasolina; enquanto que com o catalisador ADZ3 obteve-se uma quantidade mais significativa de diesel. Nos polietilenos a produção de diesel aumentou ligeiramente com a diminuição do percentual de catalisador empregado. Com apenas 10\% de ADZ3, foi possível obter uma quantidade similar de diesel à encontrada no craqueamento térmico, com maior rendimento em gasolina e redução significativa de pesados. A maior produção de diesel com o catalisador ADZ3, que possui elevado volume de mesoporos e é mais ativo, pode ser atribuída a maior difusividade dos reagentes e produtos no mesmo, em comparação com o catalisador de FCC exausto, cujos poros estão parcialmente bloqueados por coque. A maior mobilidade dos produtos de reação nos poros da ADZ3 faz com que o tempo de contato com os centros ativos seja menor, conseqüentemente o craqueamento a frações mais leves é dificultado. De uma forma geral, o craqueamento catalítico de todos os polímeros apresentou um rendimento em gasolina na faixa de 80 a $90 \%$. Desta forma, verifica-se que a seletividade dos catalisadores, como esperado, não é influenciada pelo percentual mássico empregado no processo.

\section{Conclusões}

No craqueamento catalítico de poliolefinas utilizando catalisadores zeolíticos, verifica-se a obtenção preferencial de gasolina, GLP e diesel, produtos importantes na matriz energética brasileira. $\mathrm{O}$ catalisador de FCC exausto é mais seletivo para a produção de gasolina e GLP, enquanto a produção de diesel é mais favorecida com o catalisador ADZ3, que também apresentou rendimento em gasolina elevado ( 80 a $90 \%$ ). O rendimento e a seletividade foram praticamente independentes da fração mássica de catalisador empregada no processo.

Com base nesses resultados, o emprego de catalisadores de FCC exaustos (rejeito de refinarias de petróleo) no craqueamento de poliolefinas é uma opção muito promissora para o tratamento desses rejeitos. Embora sejam necessários estu- 
dos mais específicos, os resultados ainda sugerem, que a distribuição de produtos é função não apenas da estrutura macromolecular dos polímeros como também, das características ácidas dos centros ativos, da acessibilidade e da difusividade dos reagentes e produtos dentro dos poros do catalisador. Estes fatores parecem atuar, em conjunto, na eficiência do processo de craqueamento dos polímeros estudados.

\section{Agradecimentos}

Os autores agradecem à FAPERJ pelo apoio financeiro, ao Dr. Eledir V. Sobrinho (UNIFACS -BA) pelo fornecimento do catalisador ADZ3 e ao CENPES/PETROBRAS pela doação do catalisador de FCC.

\section{Referências Bibliográficas}

1. Valle, M. L. M.; Guimarães, M. J. O. C. - "Estudo Tecnológico da Reciclagem Química de Poliolefinas, Publicação interna, EQ/UFRJ, (2002).

2. Sampaio, C. M. S. - "Estudo da Degradação Catalítica de Poliolefinas para a Obtenção de Combustíveis", Tese de Mestrado, Universidade Federal do Rio de Janeiro, Rio de Janeiro, (2003).

3. Uddin. M. A.; Koizumi, K.; Murata, K. - Polym. Degrad. Stab., 56, 37, (1997).

4. Buekens, A. G.; Huang, H. - Res. Cons. Rec., 23, 163, (1998).

5. Luo, G.; Suto, T. - Polym. Degrad. Stab., 70, 97, (2000).

6. Predel, M.; Kaminsky, W. - Polym. Degrad. Stab., 70, 373, (2000).

7. Audisio, G.; Bertini, F.; Beltrame, P. L. - Makrom. Chem. Macromol Symp., 57, 191, (1992).

8. Ohkita, H.; Nishiiiyama, R.; Tochihara, Y. - Ind. Eng. Chem. Res., 32, 3112, (1993).

9. Zhao, W.; Hasegawa, S.; Fujita, J. - Polym. Degrad. Stab., 53, 129, (1996).
10. Murty, M. V. S.; Grulke, E. A.; Bhattacharyya, D. - Polym. Degrad. Stab., 61, 421, (1998).

11. Lim, R.; White, R. L. - J. Appl. Polym. Sci., 58, 1151, (1995).

12. You, Y. S.; Shimb, J. S. - Cat. Let., 59, 221, (1999).

13. Lee, K-H.; Noh, N-S; Shin, D-H.; Seo, Y. - Polym. Degrad. Stab., 78, 539, (2002).

14. Ali, S.; Garforth, A. A.; Harris, D. H.; Rawlence, D. J.; Uemichi, Y. - Cat. Tod., 75, 247, (2002).

15. Leprince, P. - "Conversion Processes", vol. 3, cap. 3, Technip, França, (2001).

16. Sobrinho, E. V. - “ Preparação e Caracterização da Zeólita $Y$ com Alto Teor de Silício por Desaluminação em Série", Tese de Mestrado, Universidade Federal de São Carlos, São Carlos, (1997).

17. Hatakeyama, T.; Quinn, F. X. - "Thermal Analysis: Fundamentals and Application to Polymer Science, John Wiley \& Sons, England, (1994).

18. Aguado, J.; Serrano, D. P.; Escola, J. M. - Polym. Degrad. Stab., 69, 11, (2000).

19. Doyle, C. D. - J. Appl. Polym. Sci., 5, 285, (1961).

20. Wu, C. H.; Chang, C. Y.; Hor, J. L. - Pyr. Kinet. Wast. Man., 13, (1993).

21. Westerhouse, R. W.; Waanders, J.; Kuipers, J. - Ind. Eng. Chem. Res., 36, 36, (1997).

22. Fernandes, V. J.; Araújo, A S.; Fernandes, G. J. T. - J. Therm. Anal., 49, 255, (1997).

23. Shirmer, J.; Kim, J. S.; Klemm. E. - J. Anal. \& Appl. Pyr., 60, 205, (2001).

24. Walendziewski, J.; Steininger, M. - Cat. Tod., 65, 325, (2001).

Recebido: 20/06/03

Aprovado: 10/10/03 